\title{
La cirugía de aneurismas de aorta abdominal asintomáticos de entre 4 y 5,5 cm. no mejora la sobrevida
}

The UK Small Aneurysm Trial Participants. Mortality results for randomised controlled trial of early elective surgery or ultrasonographic surveillance for small aortic aneurysms. Lancet 1998; 352:1649-55

\section{Objetivo}

Comparar la cirugía de los aneurismas asintomáticos de aorta abdominal (AAA) de entre 4 y $5,5 \mathrm{~cm}$. de diámetro con la conducta expectante.

\section{Diseño}

Ensayo clínico multicéntrico randomizado y controlado. Seguimiento de 4,6 años.

\section{Lugar}

93 hospitales de Gran Bretaña.

\section{Pacientes}

Se randomizaron 1090 pacientes con AAA asintomáticos de entre 4 $5,5 \mathrm{~cm}$. a cirugía electiva ó seguimiento con ecografía. El seguimiento se realizaba cada 6 meses para los AAA de entre $4-4,9 \mathrm{~cm}$. y cada 3 para aquellos entre 5 y $5,5 \mathrm{~cm}$. Si la tasa de crecimiento excedía $1 \mathrm{~cm}$. por año, se indicaba cirugía.

\section{Medición de resultados principales}

El resultado principal era mortalidad total medida a los 30 días posteriores a la cirugía y en el seguimiento de hasta 6 años. El análisis se realizó por intención de tratar.
Resultados secundarios fueron: tasa de ruptura aneurismática y mortalidad quirúrgica.

\section{Resultados principales}

De los 563 pacientes que fueron asignados a cirugía electiva, el $87 \%$ fué operado. De los 527 pacientes asignados a seguimiento con ecografía, 206 no se operaron, 283 se operaron de acuerdo a las especificaciones del protocolo, y 38 por fuera del protocolo.

Se registraron 159 muertes en el grupo cirugía (7\%) y $150(7,4 \%)$ en el grupo observación, siendo la diferencia no significativa RR 0,94 (IC 95\% $0,74-1,19)$. Esta fué favorable al grupo observación en los primeros 6 meses, tendiendo luego a ser superado por el grupo cirugía. Se observó una tendencia no significativa favorable a la cirugía en aquellos pacientes con AAA entre 4,9-5,5 cm.

Diecisiete de los pacientes asignados a seguimiento con ecografía sufrieron ruptura aneurismática, lo que implica un riesgo del $1 \%$ anual. La mortalidad observada en la cirugía electiva a los 30 días fué del $5,8 \%$.

\section{Conclusiones}

El seguimiento con ecografía de los pacientes AAA entre 4 y $5,5 \mathrm{~cm}$. es seguro, la cirugía electiva no brinda una mejoría en la sobrevida.

\section{COMENTARIO}

Los AAA representan la décima causa de muerte en pacientes mayores, y es debida a una ruptura del mismo ${ }^{1}$. La mortalidad puede ser prevenida mediante la identificación y cirugía electiva del paciente asintomático con AAA de determinadas características de riesgo. Debe tenerse presente que la mortalidad de los pacientes con ruptura aneurismática es globalmente del $90 \%$, ya que de los que logran llegar a un hospital solo el $50 \%$ sobrevive la cirugía de urgencia ${ }^{2}$. La indicación de cirugía electiva se basa en el balance del riesgo de ruptura y, por otro lado, el de la cirugía misma. El riesgo de ruptura depende basicamente del tamaño del aneurisma y del grado de expansión del mismo ${ }^{3}$. Hasta ahora se consideraba que los AAA mayores de $5 \mathrm{~cm}$. tenían un riesgo lo suficientemente elevado como para merecer cirugía, y aquellos de entre 4 y $5 \mathrm{~cm}$. poseían un riesgo intermedio, y podían de igual manera operase o tener una conducta expectante. Esta era la recomendación de la Sociedad Americana de Cirugía Vascular y está basada en estudios retrospectivos que mostraban un riesgo global de ruptura estimada entre el 12 al 45\% en cinco años ${ }^{4}$. Este grupo reporta también una disminución sostenida de la mortalidad quirúrgica en EEUU, que era del $6 \%$ en 1986 y actualmente es entre el 2 al $4 \% 5$.

El presente estudio es un ejemplo de como un ensayo clínico controlado puede brindar claridad y nitidez, y de como basarse en estudios retrospectivos puede llevar a afirmaciones no solamente equivocadas si no también peligrosas. Los datos reportados por el estudio muestra dos elementos muy importantes para la toma de decisiones, el riesgo de ruptura es significativamente menor al que se estimaba, del $1 \%$ anual y la mortalidad de la cirugía es mucho más alta del 5,8\%, no encontrándose una diferencia significativa entre los distintos hospitales que participaron, lo que implica que no hubo una diferencia por la selección de centros de referencia. Claramente los datos reportados por los norteamericanos pro- vienen de centros altamente calificados que pueden intervenir sobre aneurismas más grandes si la mortalidad quirúrgica es menor, pero de ninguna manera se deben tomar en forma literal recomendaciones que pueden ser útiles en realidades distintas inclusive dentro de los mismos paises desarrollados (el estudio se realizó en el Reino Unido). Si en el estudio se hubiesen seguido las recomendaciones previas, se habrian operado más pacientes y la mortalidad probablemente hubiese sido mayor. Por lo tanto, una conclusión que se desprende del estudio es que el riesgo de ruptura es menor del que se pensaba y que para tomar una desición es fundamental conocer la mortalidad quirúrgica del centro en que trabajemos. No existen claramente en nuestro medio datos sobre la mortalidad quirúrgica. Los pocos que publican provienen de centros con llamativos éxitos, pero resulta a toda vista poco ético no reportar estos datos cualesquiere fuesen sus resultados, y es el motivo por el cual la evidencia que se desprende de ensayos clínicos randomizados y controlados resulta demoledora. Otra conclusión que se establece del seguimiento es que una estrategia conservadora no implica mayor riesgo, pero debe aclararse que los médicos y ecografistas en el gupo vigilancia están más estimulados para el monitoreo de complicaciones que en la práctica habitual. Esto puede explicarse por el hecho que si bién solo 17 pacientes asignados a este grupo sufrieron ruptura, el resto pudo ser detectado antes, incluyendo aquellos en los que el AAA se hizo sintomática, (esto implica que están al borde de la ruptura), cuyo número no se reporta, pero que debe haber sido lo suficientemente alta como para elevar la mortalidad quirúrgica del grupo vigilancia al $7,1 \%$.

Este estudio cambia el manejo de los AAA. Los AAA entre 4y 5,5 cm. pueden ser seguidos muy cuidadosamente con ecografía y control clínico de los síntomas, los AAA mayores de $5 \mathrm{~cm}$. podrían operarse si se lograse disminuir la mortalidad quirúrgica a cerca del $3 \%$.

Dr. Augusto Granel Unidad de Medicina Familiar y Preventiva. Hospital Italiano de Buenos Aires.

\section{Referencias}

1. Lederle F, Johnson G, Wilson S, et al. Prevalence and associations of abdominal aortic aneurysms detected through screening. Ann Intern Med 1997;126:441-449

2. Manfredini R, Portaluppi F, Grandi E, et al. Out of hospital sudden death referring to an emergency department. J Clin Epidemiol 1996; 49: 865-68

3. Nevitt MP, Ballard DJ, Havitt JW. Prognosis of abdominal aortic aneurysms: a population based study. N EngL J Med 1989: 231: 1009-14

4. Hollier LH, Taylor LM, Ochsner J. Recommended indications for operative treatment of abdominal aortic aneurysms: Report of a subcomitee of the Joint Council of the Society for

Vascular Surgery and athe North American Chapter of the International Society for Cardiovascular Surgery. J Vasc Surg 1992; 15:1046-56

5. Ernst C. Abdominal Aortic Aneurysms. N Engl J Med 1993; 328: 1167-1172 